\title{
Proposta e Adaptação de Atividades Desplugadas para o Ensino de Computação na Educação Básica
}

\author{
Ariane N. Rodrigues, Renato H. de Oliveira Lopes \\ Licenciatura em Computação - Universidade de Pernambuco (UPE) - Campus \\ Garanhuns - Pernambuco - Brasil \\ ariane.rodrigues@upe.br, renato.hill@gmail.com
}

\begin{abstract}
Initiatives that promote the teaching of fundamentals of Computer Science can be enabled with activities of the unplugged method whose philosophy is to promote playful learning without the use of technological devices. This article describes the adaptation of the activity "Counting the Points", in addition to the presentation and integration with a new activity, "Turning the Circuit". As combined activities, contents such as the binary system and Boolean logic, their representation and the relationship with the internal workings of the computer can be promoted. The results of application in basic education, in view of the learning objectives of the activities, are also presented.
\end{abstract}

Resumo. Iniciativas que fomentam o ensino de fundamentos da Ciência da Computação podem ser viabilizadas com atividades do método desplugado cuja filosofia é promover a aprendizagem lúdica sem uso de aparatos tecnológicos. Este artigo descreve a adaptação da atividade "Contando os Pontos", além da apresentação e integração com uma nova atividade, a "Ligando o Circuito". Como atividades combinadas, conteúdos como o sistema binário e a lógica booleana, sua representação e a relação com o funcionamento interno do computador podem ser promovidos. Os resultados de aplicação na educação básica, diante os objetivos de aprendizagem das atividades, também são apresentados.

\section{Introdução}

Aprender fundamentos da Ciência da Computação permite ao aluno desenvolver o raciocínio lógico, que para [Nunes 2011] é uma habilidade que auxilia no ensino de outras disciplinas da Educação Básica. Segundo [França e Amaral 2013], constantes debates acerca da implantação de um currículo de Computação no Brasil são promovidos e mesmo com um processo ainda incipiente, várias iniciativas são válidas. Pesquisas acadêmicas, práticas com a robótica, uso de softwares educativos para o ensino de programação e entre outras, são exemplos de iniciativas realizadas em escolas de várias partes do país.

Uma das principais vantagens em estimular a aprendizagem de conceitos de computação está em formar cidadãos criativos, capazes de pensar em soluções para problemas cotidianos. Para [Wing 2006], a habilidade de pensar e resolver problemas através de fundamentos da computação é definido por pensamento computacional. Os pilares do pensamento computacional consideram técnicas da computação como a 
decomposição, abstração, reconhecimento de padrões e algoritmos que podem ser assimilados através de atividades desplugadas do livro "Computer Science Unplugged (CSU)" [Bell e Whitten e Fellows 2007]. O principal argumento do método desplugado está em ensinar fundamentos da área sem a necessidade de computador. Para [Costa et al 2012], este fato permite que práticas pedagógicas sejam realizadas em diferentes estruturas escolares, independente de hardware e/ou software.

O contexto desta pesquisa envolve um processo de criação de uma atividade desplugada para facilitar o entendimento do sistema binário como conceito principal da atividade "Contando os Pontos" do livro CSU. Percebe-se que na literatura há muitos relatos de aplicação desta atividade, sobretudo, pela exposição dos resultados e desafios enfrentados. Alguns autores consideram adaptações na aplicação, mas não descrevem ou justificam com clareza a sua natureza. Uma seção de trabalhos relacionados nesta pesquisa destaca relatos de aplicações da atividade "Contando o Pontos" e as adaptações adotadas. Esta pesquisa considera a importância em reportar as adaptações realizadas para que os conteúdos abstraídos possam ser melhor compreendidos pelos alunos, assim como facilitar novas aplicações. Deve-se considerar também a possibilidade de construir novas atividades que possam agregar as atividades do livro ao estimular possibilidades de praticar os conceitos assimilados. Acredita-se que divulgar adaptações além de novas atividades desta filosofia incentiva a concepção de materiais pedagógicos para a prática de ensino em computação nas séries iniciais.

Diante este contexto, este artigo tem como objetivo apresentar uma proposta de adaptação a atividade "Contando os Pontos", além de apresentar uma nova atividade desplugada que pode ser combinada a ela. A atividade Ligando o Circuito foi concebida com o objetivo de promover a prática do conteúdo aprendido em Contando os Pontos. A recomendação é que elas possam ser combinadas na prática. Assim, os conteúdos de sistema binário e a lógica booleana são relacionados aos circuitos internos do computador através de um tabuleiro com peças que representam operadores lógicos como E (and), OU (or) e NÃO (not). Detalhes associados aos objetivos de aprendizagem da Ligando o Circuito, materiais necessários e como aplicar também são descritos neste artigo. Além disso, resultados da aplicação das atividades no $9^{\circ}$ ano, com destaque para a verificação dos objetivos de aprendizagem das atividades também é apresentado. No geral, observou-se que houve aprendizagem dos conceitos associados as atividades na aplicação e os alunos se interessaram por aprender mais fundamentos da área de Computação.

\section{Trabalhos Relacionados}

Atualmente, o uso de computadores e celulares por crianças e adolescentes é muito frequente e há quem demonstre interesse em entender o funcionamento destes aparelhos e como eles são programados. Na prática, conceitos relacionados a Ciência da Computação podem ser aprendidos de diferentes formas, inclusive com a computação desplugada e as atividades do livro CSU. Em síntese, os conteúdos abordados nas atividades do livro ilustram a representação da informação (números binários e alfabetos), ordenação e busca de dados, autômatos de estados finitos, grafos e ocorrência e situações de impasse (deadlocks) [Bell e Whitten e Fellows 2007].

Diante este contexto, esta pesquisa apresenta trabalhos relacionados como o de [Sousa et al 2011], [Costa et al 2012] e [Vieira e Passos e Barreto 2013] que apresentam 
relatos de experiência de aplicação das atividades desplugadas no ensino médio. Além disso, destacam-se os trabalhos de [Scaico et al 2012] e [Gonçalves et al 2013] que descrevem adaptações de atividades desplugadas. É importante considerar que estas pesquisas inspiram a idealização desta pesquisa, com foco na exposição das adaptações das atividades para melhorar a compreensão dos conceitos abstraídos de computação mantendo a ludicidade ao processo de aprendizagem. Em [Scaico et al 2012], descrevese a experiência da aplicação da Atividade Contando os Pontos como proposta principal do trabalho, em uma escola pública de João Pessoa - PB nas turmas do $5^{\circ}$ ao $9^{\circ}$ ano do Ensino Fundamental. $\mathrm{O}$ autor descreve como adaptação a atividade a adição de mais cartões, estes com a representação de lâmpadas acesa e apagada. Ao considerar a operacionalização da dinâmica inerente a atividade deve-se considerar que a quantidade excessiva de cartões poderia atrapalhar e sobrecarregar o esforço cognitivo dos alunos. Mesmo assim entende-se que a ideia da representação agrega ao processo de aprendizagem. E, para facilitar a dinâmica, as representações das lâmpadas poderiam vir no mesmo cartão, na frente e verso.

O trabalho de [Gonçalves et al 2013] não relata aplicação de atividades do livro CSU mas apresenta um objeto de aprendizagem criado por estudantes de Licenciatura em Computação de Minas Gerais com o propósito de ensinar conceitos dos operadores lógicos na filosofia desplugada. $\mathrm{O}$ autor destaca um jogo de cartas chamado EvOU. $\mathrm{O}$ jogo é composto por dois tabuleiros com peças de encaixe. Cada tabuleiro é dividido em três partes, onde cada um tem espaços que permitem encaixar os cartões representados pelas operações lógicas "e", "ou" e "não", além do "0" e "1". O objetivo é realizar as operações de acordo com cada tabuleiro e os cartões necessários para obter um resultado como saída. O autor não deixa claro se há desafios específicos para aplicação mas considera que o jogo é finalizado quando o aluno completa o encaixe de todo o tabuleiro. As características e estrutura deste jogo serviram como forte inspiração para a concepção da atividade Ligando o Circuito. Esta pesquisa defende a ideia de fomentar possibilidades de atividades que estimulem a capacidade de análise e interpretação dos alunos. Na Ligando o Circuito, os conceitos são representados em um circuito, como formato que referencia as placas internas de um computador. Desta maneira acredita-se que o aluno possa identificar a relevância do sistema binário, a lógica e a representação das portas lógicas o funcionamento do computador. Considerar que a divulgação das adaptações possa ajudar a evoluir atividades existentes, além de estimular práticas para o ensino de Computação, é um grande avanço para pesquisas que estimulam a qualidade de formação dos alunos da Educação Básica.

\section{Atividades Desplugadas}

\subsection{Proposta de Adaptação - Contando os Pontos}

A "Contando os Pontos" é a atividade que compõem o grupo de atividades do livro CSU que objetiva explicar a relação dos dados e a representação da informação pelos computadores. Por ser uma das mais escolhidas para aplicação, ela objetiva explicar o armazenamento e transmissão de dados com menção ao sistema binário. Em síntese, a atividade considera como material cinco cartões com pontos marcados (1, 2, 4, 8 e 16) em um lado e nada em seu verso. A sequência dos pontos representa os números na base dois como referência ao sistema de representação binária adotado pelos computadores. 
Idealmente, os alunos precisam correlacionar os sistemas de representação (decimal e binário) para entender e converter os números de uma representação para outra.

Para uma aplicação efetiva da atividade, a ordem dos cartões deve ser considerada. Na prática, percebe-se que este requisito condiciona o aluno a memorizar o posicionamento dos cartões, o que impacta negativamente na compreensão do processo de conversão. Além disso, a forma "mecânica" de responder, no sentido de dar respostas sem cogitar a lógica da representação, inviabiliza o seu propósito. Para mitigar estes desafios, esta pesquisa propõe como adaptação a esta atividade a inclusão de mais cinco cartões com a representação de lâmpadas baseada na adaptação proposta em [Scaico et al 2012]. Neste sentido, o material necessário seriam dez cartões. Cinco deles equivalente ao material original, e os demais com a representação de lâmpadas acesas e apagadas, impressas frente e verso dos cartões, conforme ilustra a Figura 1.
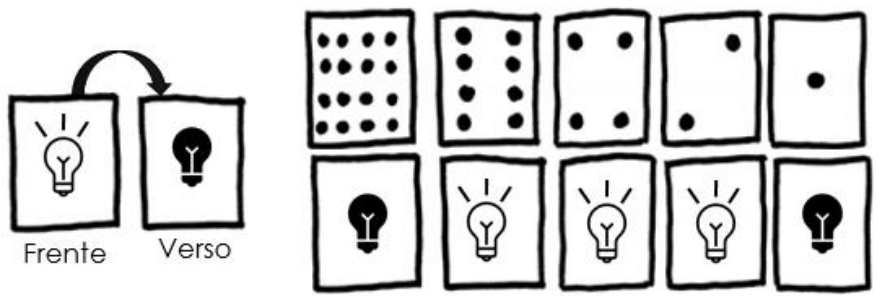

Figura 1. Proposta de adaptação de cartas com representações de lâmpadas.

Acredita-se que esta adaptação possa reforçar na representação, uma vez que os cartões das lâmpadas deverão ser usados junto aos cartões dos pontos. Recomenda-se que os cartões das lâmpadas possam ser posicionados à frente dos cartões dos pontos para que os alunos possam associar os valores 1 e 0 referente aos bits verdadeiro e falso, respectivamente para a lâmpada acesa e apagada. Esta representação, propositalmente integrada a atividade, faz referência a portas lógicas, como início para o entendimento da atividade Ligando o Circuito como nova atividade proposta na pesquisa. A próxima seção descreve com detalhes os objetivos de aprendizagem associados à atividade e seu propósito.

\subsection{Atividade Lógica - Ligando o Circuito}

A atividade Ligando o Circuito foi criada com o propósito de promover a prática dos conteúdos assimilados na atividade Contando os Pontos. Como atividades combinadas, a ligando o circuito fomenta a explicação da lógica booleana de George Boole com ênfase nos conceitos da tabela verdade dos operadores lógicos AND (e), OR (ou) e NOT (não) [Brookshear 2013]. Acredita-se que, ao adotá-la após a contando os pontos, a integração na aprendizagem pela lógica, sua representação e a relação ao funcionamento interno do computador possa ser promovida. A atividade é considerada desplugada porque segue a filosofia da Computação Desplugada sendo baseada em materiais como papel ofício A4, papelão e/ou borracha (e.v.a.). Em síntese, a atividade consiste na representação de um circuito eletrônico em forma de tabuleiro, como um jogo similar ao encaixe de peças. As peças referenciam as operações lógicas (and, or e not) contidas nos circuitos. Os destaques da Figura 2 ilustram o tabuleiro que representa o circuito (a); as peças representantes das operações com notações gráficas para as portas lógicas $\mathrm{E}, \mathrm{OU}$ e NÃ̃ (b); peça extra com função de fechar parte do circuito onde ela for inserida (c) e peças para o resultados das saídas do circuito (d). 


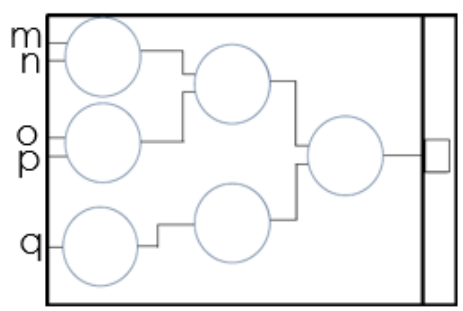

(a)

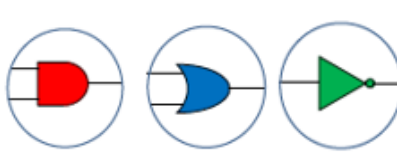

(b)

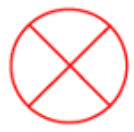

(c)

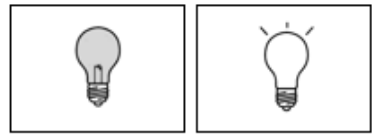

(d)

Figura 2. Materiais necessários a atividade Ligando o Circuito.

O circuito é composto por entradas (representadas pelos espaços circulares) e uma única saída (espaço retangular). Nas entradas, o aluno pode escolher e encaixar as peças dos operadores lógicos (b) e analisar o resultado das operações e indicar a saída com as peças das lâmpadas (d). Assim como a proposta para a atividade Contando os Pontos que considera a adaptação da representação com as lâmpadas, esta representação no circuito indica os resultados das operações lógicas associados aos valores 0 e 1 ao indicar ausência/presença de energia. Para auxiliar a compreensão da lógica das combinações dos operadores, um material de apoio foi criado com as tabelas verdades, adaptadas com a representação das lâmpadas e das portas lógicas.

Toda atividade desplugada possui objetivos de aprendizagem que consideram habilidades cognitivas que os alunos podem adquirir com sua aplicação. Os objetivos de aprendizagem da Ligando o Circuito foram definidos tendo como base a taxonomia de Bloom revisada [Krathwohl 2002] e pela estrutura de verbos e substantivos proposta em [Ferraz e Belhot 2010] e [Airasian e Miranda 2002]. Em síntese, os objetivos para esta atividade consideram quase todos os processos cognitivos da taxonomia (lembrar, entender, aplicar, analisar e avaliar). O único processo que não se relaciona é o criar porque a atividade não oferece a possibilidade do aluno criar algo novo diante os conteúdos relacionados às atividades. A Tabela 1 apresenta os enunciados de cada objetivo e sua relação com a categoria do conhecimento.

Tabela 1. Objetivos de aprendizagem da atividade Ligando o Circuito.

\begin{tabular}{|c|l|c|}
\hline Objetivos & \multicolumn{1}{|c|}{ Enunciados } & $\begin{array}{c}\text { Processo do } \\
\text { Conhecimento }\end{array}$ \\
\hline OA1 & $\begin{array}{l}\text { Entender o armazenamento de dados do computador explicando o sistema } \\
\text { binário. }\end{array}$ & Conceitual \\
\hline OA2 & $\begin{array}{l}\text { Analisar e lembrar do funcionamento do sistema binário implementando a } \\
\text { conversão de números binários em decimal e vice-versa presente na } \\
\text { atividade "Contando os Pontos". }\end{array}$ & $\begin{array}{c}\text { Efetivo e } \\
\text { Procedural }\end{array}$ \\
\hline OA3 & $\begin{array}{l}\text { Entender o funcionamento de interpretação de dados pelo computador } \\
\text { interpretando os operadores lógicos básicos utilizados no circuito. }\end{array}$ & Conceitual \\
\hline OA4 & $\begin{array}{l}\text { Lembrar e aplicar as operações lógicas básicas reconhecendo as tabelas } \\
\text { verdades e representações gráficas dessas operações presentes na } \\
\text { atividade "Ligando o Circuito". }\end{array}$ & Efetivo \\
\hline OA5 & $\begin{array}{l}\text { Analisar, aplicar e avaliar os resultados das operações lógicas concluindo } \\
\text { a dinâmica do circuito apresentado na atividade "Ligando o circuito". }\end{array}$ & $\begin{array}{c}\text { Procedural e } \\
\text { Metacognitivo }\end{array}$ \\
\hline
\end{tabular}

Pode-se destacar que os objetivos OE2, OE4 e OE5 combinam mais de um processo cognitivo. Esta flexibilidade, inerente a taxonomia revisada, favorece o estímulo a diferentes processos cognitivos simultaneamente durante a aprendizagem. Os processos do conhecimento, quando mapeados aos objetivos, relacionam o tipo de 
conhecimento a ser adquirido ao processo cognitivo. Neste caso, o OE1 mapeado ao processo conceitual considera que o aluno precisa compreender conceitos inerentes ao armazenamento de dados através do sistema binário. No OE2, os processos efetivo e procedural consideram que os alunos precisam lembrar de detalhes do sistema binário para utilizar a técnica de conversão, respectivamente. Por sua vez, o OE3, mapeado ao processo conceitual, os alunos precisam entender e interpretar cada operação para posteriormente aplicar a lógica no circuito. Neste sentido, o OE4 considera o processo efetivo porque é necessário que o aluno lembre da associação entre as operações booleanas e suas notações gráficas, além dos valores binários (0 ou 1) para lâmpada acesa ou apagada. Por fim, o OE5 quando mapeado aos processos procedural e metacognitivo, reforçam a prática pela combinação das operações lógicas do circuito, que cabe ao aluno avaliar o contexto do circuito diante o posicionamento das peças e indicar o resultado corretamente. Este último objetivo também considera que o aluno precisa definir suas próprias estratégias metacognitivas ao escolher as peças que o levem a alcançar os desafios do circuito. É importante considerar que esta atividade estimula a liberdade de escolha, pois, diferentes possibilidades de encaixe podem ser idealizadas pelos alunos a cada desafio. Os desafios, os materiais necessários, o tempo estimado para aplicação, e outros aspectos foram descritos em um guia baseado no modelo de apresentação das atividades do livro CSU. A próxima seção apresenta alguns aspectos dele.

\subsection{Guia para aplicação das atividades}

Qualquer pessoa que tenha interesse em aplicar as atividades desplugadas encontra no guia detalhes que o oriente na execução. Idealmente, ele foi criado para o professor ou licenciado em computação porque indica ações para a execução como pela promoção de discussões e forma de avaliação alinhada aos objetivos de aprendizagem.

Por recomendação, a atividade Ligando o Circuito pode ser aplicada em duplas ou trios (a depender da quantidade da turma). O material necessário para cada dupla/trio são: circuito, grupo de peças (portas lógicas (6), função extra (1) e saída (2)) e guia de tabelas verdades. Esta atividade deve ser apresentada após a Contando os Pontos adaptada. Para que os alunos possam colocar em prática o raciocínio lógico, três desafios devem ser apresentados. A Tabela 2 apresenta a descrição dos desafios, assim como as possíveis discussões que podem ser idealizadas durante o jogo.

Tabela 2. Desafios da atividade Ligando o Circuito.

\begin{tabular}{|c|l|l|}
\hline Desafios & \multicolumn{1}{|c|}{ Descrições } & \multicolumn{1}{|c|}{ Discussões } \\
\hline 01 & $\begin{array}{l}\text { Considere os valores (m, n, o, p, q) de entrada das portas } \\
\text { lógicas do circuito. Encaixe os operadores lógicos para } \\
\text { que o resultado de saída seja lâmpada acesa. }\end{array}$ & $\begin{array}{l}\text { Há diferenças na quantidade } \\
\text { de } \\
\text { entradas nos operadores } \\
\text { lógicos. O aluno precisa } \\
\text { perceber que as portas and e } \\
\text { or possuem duas entradas, } \\
\text { enquanto que a not possui } \\
\text { apenas uma. }\end{array}$ \\
\hline 03 & $\begin{array}{l}\text { Considere os valores (m, n, o, p, q) de entrada das portas do circuito. Encaixe os operadores lógicos para } \\
\text { que o resultado de saída seja lâmpada apagada. }\end{array}$ & $\begin{array}{l}\text { A mudança do operador } \\
\text { lógico influencia diretamente } \\
\text { no resultado do circuito. }\end{array}$ \\
\hline
\end{tabular}


O professor precisa definir os valores de entrada do circuito (destaque (a) figura 2). Como exemplo, os valores $(1,0,0,0,1)$ servem para que o aluno consiga analisar as possibilidades de encaixe das portas lógicas para obter os resultados corretos a cada desafio. Para cada desafio, discussões podem ser levantadas e esclarecidas durante a aplicação. Como avaliação, pode-se observar e verificar se os desafios foram resolvidos, considerando os objetivos 3, 4 e 5. Além disso, o guia também indica um questionário com perguntas que investigam o entendimento dos alunos com referência aos objetivos de aprendizagem das atividades. A próxima seção apresenta detalhes da aplicação com destaque para os resultados.

\section{Aplicação das atividades}

Quatro atividades básicas foram definidas para guiar a aplicação das atividades desplugadas. A Tabela 3 sintetiza as atividades e as tarefas associadas a cada uma delas.

Tabela 3. Atividades e tarefas estabelecidas para aplicação das atividades deslugadas.

\begin{tabular}{|c|c|}
\hline Atividades & Tarefas \\
\hline Preparação & $\begin{array}{c}\text { - Preparar os materiais necessários as atividades; definir e elaborar o questionário } \\
\text { para avaliação de aprendizagem e; executar um teste piloto. }\end{array}$ \\
\hline Execução & $\begin{array}{c}\text { - Explicar e conduzir a dinâmica de aplicação pela sequência das atividades } \\
\text { desplugadas; observar e registrar o desempenho dos participantes a cada desafio } \\
\text { e; aplicar o questionário. }\end{array}$ \\
\hline Interpretação & - Reunir e sumarizar os dados coletados com o questionário. \\
\hline $\begin{array}{c}\text { Consolidação } \\
\text { dos Resultados }\end{array}$ & $\begin{array}{l}\text { - Relatar os resultados obtidos, sob a ótica das respostas do questionário alinhadas } \\
\text { aos registros da execução. }\end{array}$ \\
\hline
\end{tabular}

A etapa de preparação é essencial porque envolve um planejamento necessário para a condução da aplicação. Neste caso, todos materiais foram impressos e organizados diante a quantidade de participantes considerados para um teste piloto antes da aplicação principal. Um questionário foi elaborado com nove assertivas baseadas nos objetivos de aprendizagem das atividades para obter informações acerca da aprendizagem dos participantes após a aplicação. Para cada assertiva, o participante deveria indicar "Concordo", "Indeciso" ou Discordo". Duas perguntas abertas também foram consideradas para obter opinião sobre a contribuição das atividades para a aprendizagem em computação. Além disso, o grau de satisfação ao aprender com as atividades, em uma escala de (0 nenhuma - 5 muita) também foi um aspecto questionado. O piloto foi executado com vinte estudantes do primeiro período do curso de Licenciatura em Computação da Universidade de Pernambuco com o intuito de avaliar a dinâmica provida pelos materiais das atividades e seu impacto sobre a aprendizagem junto ao instrumento de avaliação. A participação dos estudantes foi efetiva, e por eles ainda não possuírem o conhecimento a respeito dos conteúdos abordados, acredita-se que não houve influências que inviabilize a aplicação. Com a aplicação piloto, informações relevantes foram identificadas, como o tempo necessário para a aplicação das atividades (média de 2h) e o nível de dificuldades dos desafios. Além disso, alguns ajustes nas peças também foram considerados para a execução principal da aplicação.

Em relação a aplicação principal, as atividades foram aplicadas com vinte alunos do $9^{\circ}$ ano do ensino fundamental da Escola Professora Ivonita Alves Guerra, com 
duração média de duas horas. A escolha do ano considera a faixa etária recomendada pelas próprias atividades desplugadas e a seleção dos participantes foi aleatória. Foi utilizado o recurso datashow apenas para a apresentação das definições e informações acerca dos conteúdos necessários à aplicação (sistema binário e operações booleanas). Primeiro a atividade adaptada Contando os Pontos foi executada, em seguida, Ligando o Circuito. Os participantes mostraram interesse e participaram ativamente das duas atividades. Perguntas a respeito da interpretação dos desafios a serem realizados foram sendo esclarecidas ao longo do processo. A execução foi conduzida por apenas um licenciado, com experiência em sala de aula, provida pelas práticas e estágios.

\section{Resultados}

Esta seção apresenta os resultados de aprendizagem identificados pela aplicação principal das atividades desplugadas. Durante a execução, observou-se a participação com o intuito de verificar se os desafios foram resolvidos. Em alinhamento a esta percepção, os resultados do questionário indicam quais os objetivos de aprendizagem foram adquiridos, sob a ótica dos participantes.

A aplicação da atividade Ligando o Circuito foi realizada em duplas, totalizando dez duplas. No geral, dos três desafios, apenas duas duplas não conseguiram concluir apenas o primeiro desafio. Atribui-se este resultado a dificuldade inicial em compreender a lógica associada aos operadores. Observou-se também que a boa interação entre as duplas proporcionou uma aprendizagem discursiva entre eles. Para sintetizar os resultados, a Tabela 4 apresenta a relação das assertivas do questionário aos objetivos e o percentual para cada opção de resposta (Concordo (C), Indeciso (I) e Discordo (D)).

Tabela 4. Resultados das assertivas do questionário de avaliação.

\begin{tabular}{|c|c|c|c|c|}
\hline \multicolumn{2}{|r|}{ Resultados } & \multicolumn{3}{|c|}{ Percentuais } \\
\hline Objetivos & Assertivas & $\mathrm{C}$ & I & D \\
\hline \multirow{3}{*}{ OE1 e OE2 } & $\begin{array}{l}\text { Consegui associar a representação da lâmpada a sua função na } \\
\text { atividade Contando os Pontos. }\end{array}$ & $100 \%$ & $0 \%$ & $0 \%$ \\
\hline & $\begin{array}{l}\text { Depois da atividade Contando os Pontos consigo converter um } \\
\text { número binário em decimal, assim como um decimal em binário. }\end{array}$ & $100 \%$ & $0 \%$ & $0 \%$ \\
\hline & $\begin{array}{l}\text { Depois da atividade Contando os Pontos consegui entender e } \\
\text { explicar o sistema binário e o armazenamento de dados do } \\
\text { computador. }\end{array}$ & $80 \%$ & $20 \%$ & $0 \%$ \\
\hline \multirow{3}{*}{ OE3 e OE4 } & $\begin{array}{l}\text { É fácil diferenciar as portas lógicas pela representação simbólica } \\
\text { utilizada na atividade de Ligando o Circuito. }\end{array}$ & $60 \%$ & $30 \%$ & $10 \%$ \\
\hline & $\begin{array}{l}\text { É difícil entender a lógica dos operadores usados na atividade } \\
\text { Ligando o Circuito. }\end{array}$ & $30 \%$ & $25 \%$ & $45 \%$ \\
\hline & $\begin{array}{l}\text { Entendi a lógica das operações booleanas e conseguiria analisar o } \\
\text { resultado em outro circuito. }\end{array}$ & $45 \%$ & $35 \%$ & $20 \%$ \\
\hline \multirow[t]{2}{*}{ OE5 } & $\begin{array}{l}\text { Entendi os conceitos booleanos após usar o circuito e a atividade } \\
\text { contando os pontos. }\end{array}$ & $75 \%$ & $25 \%$ & $0 \%$ \\
\hline & Achei os desafios da atividade Ligando o Circuito muito difíceis. & $20 \%$ & $30 \%$ & $50 \%$ \\
\hline
\end{tabular}

Com os resultados expostos pela Tabela, pode-se considerar que a aprendizagem foi satisfatória. Em síntese, os alunos conseguiram atingir os processos cognitivos estabelecidos como objetivos das atividades. Percebeu-se que houve aprendizagem pela compreensão da ideia da representação da lâmpada e sua relação com a conversão do 
sistema binário. Em relação ao reconhecimento das representações associadas às portas lógicas, percebeu-se que a maioria conseguiu diferenciá-las. Apesar disso, o que deve ser considerado é a interpretação necessária para realizar as operações nos desafios. Entender a lógica das operações é essencial e mesmo com apoio do material das tabelas verdade, alguns alunos apresentaram dificuldades neste aspecto. Em relação ao grau de satisfação em aprender com as atividades, pela escala de 0 a 5, obteve-se respostas para $3(20 \%), 4(35 \%)$ e $5(45 \%)$. Isto demonstra que houve forte aceitação e satisfação com a dinâmica. Atribui-se a este resultado, a atratividade inerente às atividades que tendem a prender a atenção e estimular o aluno a pensar para resolver os desafios semelhante a jogos de lógica e quebra-cabeça.

\section{Conclusões}

Esta pesquisa apresenta a adaptação e integração de atividades desplugadas como incentivo ao ensino de Computação na educação básica. A proposta de adaptação da "Contando os Pontos" considera a representação das lâmpadas acesa e apagada como referência aos bits 1 e 0 , verdadeiro e falso. Por sua vez, a atividade Ligando o Circuito considera a possibilidade prática de aplicar os conteúdos assimilados na contando os pontos de forma lúdica referente ao jogo de tabuleiro. Esta atividade enfatiza a lógica booleana associada aos circuitos e funcionamento interno do computador como pelo armazenamento e transmissão de dados. Como materiais de cunho pedagógico ao fomento do pensamento computacional, pode-se considerar a validade das atividades diante os resultados obtidos pela aplicação. Pode-se considerar que a atividade relaciona a técnica de abstração ao ignorar detalhes internos específicos aos componentes do processador. A atividade simula um circuito, logo, uma representação (ideia abstraída) de como as operações lógicas são organizadas nele. $\mathrm{O}$ aluno pode analisar os resultados das operações lógicas e assim entender melhor a lógica adotada pelo computador para processar as informações. Esta representação reforça e alinha a aplicação do sistema binário. Como próximos passos, pretende-se considerar alterações a atividade Ligando o Circuito como modificações do circuito pela adição de novas entradas e possibilidade de uso de mais portas lógicas, além de novos desafios de diferentes níveis de dificuldade, para assim dinamizar ainda mais o processo de aprendizagem em novas aplicações.

\section{Referências}

Airasian, P. W. e Miranda, H. (2002) "The Role of Assessment in the Revised Taxonomy".

Bell, T. e Whitten, I. e Fellows, M. (2007) "Computer Science Unplugged". Universidade de Canterbury, Nova Zelândia. Disponível em: http://csunplugged.org.

Brookshear, J. G. (2013) Ciência da Computação Uma visão Abrangente. $11^{\circ}$ edição, Editora Bookman.

Costa, T. e Batista, T. e Maia, M. e Almeida, L. e Farias, A. (2012) “Trabalhando Fundamentos de Computação no Nível Fundamental: experiência de licenciandos em Computação da Universidade Federal da Paraíba". In: XX Workshop de Educação em Computação (WEI’2012). Curitiba, PR, Brasil. 
Ferraz, A. P. do C. M. e Belhot, R. V. (2010) "Taxonomia de Bloom: revisão teórica e apresentação das adequações do instrumento para definição de objetivos instrucionais". Gest. Prod., São Carlos, v. 17.

França, R. S. de, Amaral, H. J. C. do. (2013) "Ensino de Computação na Educação Básica no Brasil: Um Mapeamento Sistemático”. In: XXI Workshop sobre Educação em Computação.

Gonçalves, D. A. S. e Silva, G. M. da e Luz, R. S. da e Silva, E. P. (2013) "Relato de experiência de alunos do curso de Licenciatura em Computação do IFMG-campus Ouro Branco na utilização de objetos de aprendizagem desplugados e do Scratch como instrumentos no ensino de programação". In: Anais dos Workshops do Congresso Brasileiro de Informática na Educação.

Krathwohl, D. R. (2002) "A revision of Bloom's taxonomy: An overview. Theory into practice" v. 41, n. 4, p. 212-218.

Nunes, D. J. (2011) "Ciência da computação na educação básica" In: Jornal da Ciência, v. 9.

Scaico, P. D. e Henrique, M. S. e Cunha, F. O. M. e Alencar, Y. M. de (2012) "Um Relato de Experiências de Estagiários da Licenciatura em Computação com o Ensino de Computação para Crianças”. RENOTE, v. 10, n. 3.

Sousa, R. V. e Barreto, L. P. e Andrade, A. e Abdalla, D. (2011) "Ensinando e aprendendo conceitos sobre ciência da computação sem o uso do computador: Computação Unplugged!!” Jornada de Atualização em Informática na Educação, v. 1, n. 1 .

Vieira, A. Passos, O. Barreto, R. (2013) Um Um Relato de Experiência do Uso da Técnica Computação Desplugada.

Wing, J. M. (2006) "Computational thinking”. Communications of the ACM, v. 49. 